\title{
Study of neutron fundamental properties in the perfect crystal optics and diffraction
}

\author{
V.V. Fedorov ${ }^{1,2,3, a}$ and V.V. Voronin ${ }^{1,2,3, b}$ \\ ${ }^{1}$ Petersburg Nuclear Physics Institute, National Research Center Kurchatov Institute, Gatchina 188300, Russia \\ ${ }^{2}$ Peter the Great St.Petersburg Polytechnic University, Saint-Petersburg 195251, Russia \\ ${ }^{3}$ Saint-Petersburg State University, Saint-Petersburg 199034, Russia
}

\begin{abstract}
Review of the new crystal-diffraction methods to study fundamental neutron properties and interactions is presented. Features of neutron optics in the crystal without a center of symmetry is discussed as well as the first experimental results and perspectives to improve the sensitivity of experiments on a search for the neutron electric dipole moment (EDM) and CP-violating short-range pseudomagnetic nucleon-nucleon interaction. Also, the possibility to develop ultra-precise neutron spectroscopy using Laue diffraction in the perfect crystal is discussed, which can be used for a broad range of experiments: search for the neutron electric charge; test of the equivalence principle; precise measurements of neutron scattering amplitudes; crystal quality testing.
\end{abstract}

\section{Introduction}

Neutron optics of perfect crystal is a very promising technique to study the fundamental neutron interaction with the matter. Here we discuss two approaches: the first concerns with the use of some features of neutron optics and diffraction in non-centrosymmetric crystals, the second one with the use of fine effects in Laue neutron diffraction, which allow building an ultra-precise technique to measure small variations of neutron energy and/or direction.

\section{Non-centrosymmetric crystal and neutron EDM}

The electric dipole moment of the neutron (nEDM) is one of the very sensitive probes for $\mathrm{CP}$ violation beyond the Standard Model of particle physics [1]. The importance of nEDM searches is additionally highlighted by an additional possibility to reach understanding the nature of baryon-antibaryon asymmetry in the Universe that is one of the most exciting puzzles in modern physics. The most precise experiments today use Ramseys magnetic resonance method and ultracold neutrons (UCNs) [2,3]. Further progress is presently limited by systematics [4] and the low density of UCNs available. Here we discuss an alternative approach based on a neutron spin rotation in non-centrosymmetric crystals.

The statistical sensitivity of any experiments to measure the EDM is determined by the product $E \tau N^{1 / 2}$ where $\tau$ is the duration of the neutron interaction with the electric field $E$ and $N$ the number of the counted neutrons. New projects to measure the nEDM with UCNs aim to increase the UCN density and thus $N$ by orders

\footnotetext{
a e-mail: fedorov_vv@pnpi.nrcki.ru

b e-mail: voronin_vv@pnpi.nrcki.ru
}

of magnitude [5-13]. Another possibility to improve the Ramsey's method sensitivity, using high peak flux and the time structure of a pulsed neutron beam (instead of UCN) at the planned European Spallation Source, is discussed in Ref. [14]. See also Refs. [15,16] for a recent overview. In contrast, experiments with crystals exploit the electric field inside matter, which for some crystals can be by a few orders of magnitude higher than the electric field achievable in magnetic resonance methods.

EDM experiments with absorbing crystals were pioneered by Shull and Nathans [17]. Their experiment was based on the interference of the neutron electromagnetic scattering amplitude with the imaginary part of the nuclear one. Yu. Above with his colleagues [18] were the first who paid attention to the presence of a spin-dependent term due to the interference of nuclear and spin-orbit parts of the structure amplitude in the interaction of neutrons with a non-centrosymmetric non-absorptive crystal. Such possibility of arising a spin-dependent term in a nonabsorbing crystal because of the interference between the nuclear and electromagnetic structural amplitudes was rediscovered two times: in 1983 by Forte [19] and in 1985 by Baryshevskii and Cherepitsa [20,21]. A neutron spin rotation in non-centrosymmetric crystals due to such interference as a way to search for a nEDM was considered [19-21].

In our papers Refs. [22,23] we have shown that the interference of the nuclear and the electromagnetic parts of the structure amplitude leads to a constant strong electric field (up to $10^{9} \mathrm{~V} / \mathrm{cm}$ ), acting on a neutron during all time of its movement in the noncentrosymmetric crystal. This field was measured first in a Laue geometry diffraction experiment [23], in agreement with the calculated value. Forte and Zeyen [24] attempt to test experimentally an interference of nuclear and spinorbit structure amplitudes by measuring a spin rotation angle in a non-centrosymmetric crystal, but their measured 
result turned out to be a few times less than the estimated one. Authors [24] assumed that the cause was the crystal imperfection.

\subsection{Neutron diffraction in a crystal without a centre of symmetry}

On the basis of the picture of strong electric fields, acting on neutron in a crystal without a centre of symmetry, it proved to be possible to predict and describe quite straightforwardly a number of new effects in dynamic neutron diffraction and in neutron optics. The values of these interplanar fields depend on a momentum direction and energy of neutron in crystal reaching maxima in vicinities of the exact Bragg directions or energies. Such fields give rise for new polarization phenomena $[23,25-33]$ in the neutron Laue diffraction [23, 25-30] and in the neutron optics [30-33] and provide new methods of a search for the neutron electric dipole moment (EDM) [25, $27,31,32]$. Measurement, for instance, of a spin precession angle for neutron, moving close to the Bragg condition through a thick crystal in such a strong electric field gives hope to improve the limits on the neutron EDM, achieved with the magnetic resonance method using ultracold neutrons (UCN method).

How do strong electric fields acting on a neutron in a non-centrosymmetric crystal arise? It should be noted first of all that the wave function of a diffracting neutron in a nonmagnetic crystal (as well as neutron-density distribution in the crystal) is determined exclusively by nuclear interaction. Thus, the periodic nuclear potential forms the diffraction pattern, while an electromagnetic interaction affects only the neutron spin (magnetic moment and EDM, which are both proportional to spin) without changing the spatial distribution of neutrons in the crystal.

Any potential of neutron interaction with a crystal (nuclear, electric, and so on) can be represented in the form of an expansion in the reciprocal-lattice vectors $\mathbf{g}$ as

$$
\begin{aligned}
V(\mathbf{r}) & =\sum_{a} V_{a}\left(\mathbf{r}-\mathbf{r}_{a}\right)=\sum_{g} V_{g} e^{i \mathbf{g r}} \\
& =V_{0}+\sum_{g} 2 v_{g} \cos \left(\mathbf{g r}+\phi_{g}\right),
\end{aligned}
$$

where $V_{a}\left(\mathbf{r}-\mathbf{r}_{\mathbf{a}}\right)$ is the potential of an individual atom; $\mathbf{r}_{\mathbf{a}}$ is the atom position; $V_{g}=v_{g} \exp \left(i \phi_{g}\right)$; and $g=2 \pi / d$, where $d$ is the interplanar spacing. For the sake of simplicity, we disregard here absorption, in this case $V_{g}=$ $V_{-g}^{*}$. The amplitudes for $g$ harmonics are related to the total crystal potential by the equation

$$
V_{g}=\int_{V=1} d^{3} r e^{-i \mathbf{g r}} V(\mathbf{r})=-\frac{4 \pi N_{c}}{k^{2}} F_{g}
$$

Here $k$ is a neutron wave vector, $N_{c}$ is a density of crystal cells, $F_{g}$ is a neutron scattering structure amplitude.

In general, the potential of neutron interaction with a crystal is the sum of all possible potentials: the nuclear potential $V^{N}(\mathbf{r})$ determined by distribution of the Fermi pseudopotentials (scattering lengths for neutrons interacting with nuclei of crystal atoms), the electric potential $V^{E}(\mathbf{r})$ determined by distribution of charge in crystal, and the pseudomagnetic potential $V^{S P}(\mathbf{r})$ induced by the exchange of a light pseudoscalar (axion-like) particle between a neutron and intranuclear nucleons. That is determined by distribution of a number of nucleons in crystal, etc.; that is

$$
V(\mathbf{r})=V^{N}(\mathbf{r})+V^{E}(\mathbf{r})+V^{C P}(\mathbf{r})+\ldots .
$$

We should notice that the electromagnetic interaction of neutron with crystal is determined by potential $V^{E M}(\mathbf{r})$ associated with the Schwinger interaction of the magnetic moment of a moving neutron and its EDM with electric field $\mathbf{E}(\mathbf{r})=-\nabla V^{E}(\mathbf{r})$ (not by potential $V^{E}(\mathbf{r})$ itself).

For centrosymmetric crystal, by choosing a center of symmetry as the origin of coordinates, one can reduce to zero the phases of amplitudes of all harmonics of the total potential (and of each potential individually): $\phi_{g}=\phi_{g}^{N}=$ $\phi_{g}^{E} \equiv 0$, thereby, make all harmonic amplitudes realvalued (with the result that all potentials become purely cosine). But for non-centrosymmetric crystals, there arises a nonzero phase shift between harmonics of potentials that belong to different types.

The electric field inside the crystal created by the system of crystallographic planes, which is characterized by the reciprocal-lattice vector $\mathbf{g}$, is determined by the electric potential of this system of planes; that is,

$$
\mathbf{E}(\mathbf{r})=-\operatorname{grad} V_{g}^{E}(\mathbf{r})=2 v_{g}^{E} \mathbf{g} \sin \left(\mathbf{g r}+\phi_{g}^{E}\right),
$$

where $v_{g}^{E}$ and $\phi_{g}^{E}$ are, respectively, the amplitude and phase of the $g$ harmonic of the crystal electric potential. Hereafter, we will choose the origin of coordinates at the maximum of the nuclear potential harmonic; that is, $\phi_{g}^{N}=0$. In non-centrosymmetric crystals, for some planes $\phi_{g}^{E} \neq 0$, and the positions of the maxima of electric and nuclear potentials are not coincident in space and are different for different $\mathbf{g}$. We emphasize once again that namely the nuclear potential determines the propagation of neutrons in a crystal, so that the structure of the neutron wave function replicates the structure of the nuclear potential - i.e. the positions of the maxima and minima of the squared modulus of this function coincide with the positions of the maxima and minima of $V^{N}(\mathbf{r})$. Therefore neutrons will concentrate at minima (or maxima) of nuclear potential, and so will be in a strong electric field.

It is easy to understand how such neutron concentration arises. In the first order of perturbation theory, the wave function for a neutron in a crystal under conditions close to Bragg's one can be represented in the form [33]:

$$
\psi(\mathbf{r})=e^{i \mathbf{k r}}+\frac{V_{g}^{N}}{E_{k}-E_{k_{g}}} e^{i \mathbf{k}_{g} \mathbf{r}} \equiv e^{i \mathbf{k r}}\left[1-\frac{U_{g}^{N}}{2 \Delta_{g}} e^{i \mathbf{g r}}\right] .
$$

Here, the phase of $V_{g}^{N}$ is zero; $\mathbf{k}_{g}=\mathbf{k}+\mathbf{g} ; E_{k}=$ $\hbar^{2} k^{2} / 2 m ; E_{k_{g}}=\hbar^{2} k_{g}^{2} / 2 m ; V_{g}^{N}=\hbar^{2} U_{g}^{N} / 2 m ; \Delta_{g}=\left(k_{g}^{2}-\right.$ $\left.k^{2}\right) / 2$ is a parameter that characterizes the deviation from Bragg's conditions; and

$$
|\psi(\mathbf{r})|^{2}=1-\frac{U_{g}^{N}}{\Delta_{g}} \cos (\mathbf{g r}) .
$$

For a non-centrosymmetric crystal, a nonzero electric field arises in the region of the maximum neutron concentration - that is, in the region of maxima (or minima) of $|\psi(\mathbf{r})|^{2}$ because of the shift of the electric 
potential maxima with respect to the nuclear-potential ones. The strength of the field acting on neutrons can be determined by averaging (3) over the state (4); that gives

$$
\mathbf{E}=\langle\psi|\mathbf{E}(\mathbf{r})| \psi\rangle=\frac{U_{g}^{N}}{\Delta_{g}} v_{g}^{E} \mathbf{g} \sin \phi_{g}^{E} \equiv \mathbf{E}_{g} \frac{U_{g}^{N}}{\Delta_{g}} \equiv \mathbf{E}_{g} \Delta_{B},
$$

where $\Delta_{B} \equiv U_{g}^{N} / \Delta_{g}$, is a dimensionless parameter that characterizes the deviation from the Bragg condition, $\mathbf{E}_{g}=v_{g}^{E} \mathbf{g} \sin \phi_{g}^{E}$. Expression (6) is written for the case of $\Delta_{B} \ll 1$. An accurate calculation of the electric field strength in the two-wave approximation yields

$$
\mathbf{E}=\mathbf{E}_{g} \cdot \Delta_{B} / \sqrt{1+\Delta_{B}^{2}}
$$

The presence of a neutron EDM leads to spin rotation around the reciprocal lattice vector $\mathbf{g} \| \mathbf{E}$ by the angle

$$
\varphi_{d}=\frac{2 d_{n} E}{\hbar} \tau \text {. }
$$

Here, $\tau$ is the time a neutron spends in the crystal.

It is noteworthy that the main false effects, due to the Schwinger interaction of moving neutrons with an electric field, tend to zero in the case of diffraction at the angle of $\pi / 2$ since the neutron velocity is nearly parallel to the electric field. The residual effects do not exceed in magnitude similar effects for ultracold neutrons. Moreover, the axis of neutron-spin rotation owing to the Schwinger interaction is strictly orthogonal to the electric field by virtue of its definition: $\mathbf{H}^{S}=\left[\mathbf{E}_{g} \times \mathbf{v}\right] / c$. This circumstance makes it possible to separate these effects by using the system for three-dimensional analysis of polarization [34].

The similar spin structure has $C P$-violating pseudomagnetic interaction [35]. For the $g$ harmonic amplitude of the crystal potential for such interaction one can obtain $[32,36]$

$$
V_{g}^{S P}=-i F_{g}^{S P} e^{i \Phi_{g}^{S P}} \frac{\hbar^{2} g_{S} g_{P}}{2 m V_{c}} \frac{g \lambda_{A}^{2}}{1+g^{2} \lambda_{A}^{2}}\left(\boldsymbol{\sigma} \mathbf{n}_{g}\right) \equiv \boldsymbol{\sigma} \mathbf{H}^{S P},
$$

where $g_{S}$ and $g_{P}$ are the dimensionless coupling constants for scalar and pseudoscalar neutron interactions with intranuclear nucleons and $\lambda_{A}=\hbar / m_{A} c$ is the force range (the Compton wavelength of the interaction mediator - for example, the axion), $\mathbf{n}_{g} \equiv \mathbf{g} / g, \mathbf{H}^{S P}$ is the pseudomagnetic field, while $F_{g}^{S P}$ and $\Phi_{g}^{S P}$ are, respectively, the amplitude and the phase of the structure factor $f_{g}^{S P}$ for the system of planes in question. This structure factor has the form

$$
f_{g}^{S P}=F_{g}^{S P} e^{i \Phi_{g}^{S P}}=\sum_{i} A_{i} \cdot e^{i \mathbf{g r}_{i}},
$$

where $A_{i}$ and $\mathbf{r}_{i}$ are, respectively, the mass number and the position of the $i^{\text {th }}$ atom in the unit cell. The presence of pseudomagnetic potential leads to additional spin rotation around the reciprocal-lattice vector $\mathbf{g} \| \mathbf{H}^{S P}$ by the angle

$$
\varphi_{S P}=\frac{2 V_{S P}}{\hbar} \tau,
$$

where $V_{S P}=V_{g}^{S P} \cdot \Delta_{B}$, see (6) or (7). We should notice that both effects due to EDM and pseudomagnetic potential are false to each other. But we can separate them, using for example two different plane systems for measurements.

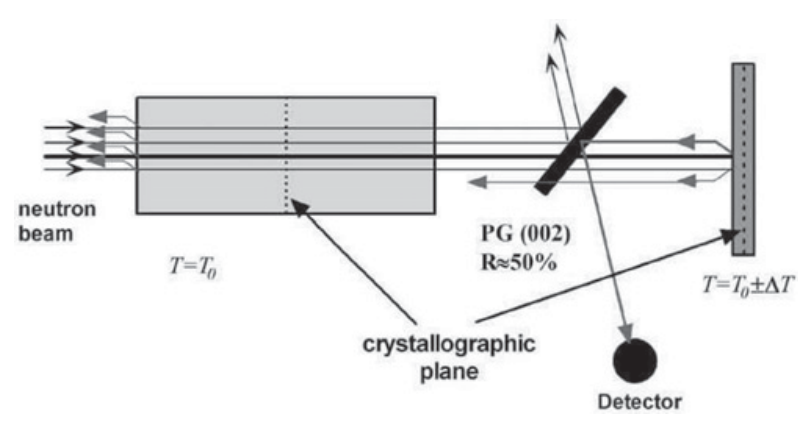

Figure 1. Scheme of the experiment to search for the neutron $\operatorname{EDM}[37,38]$.

\section{Experiments on a search for neutron EDM and CP-violating forces}

\subsection{Experimental setup}

The layout of the experiment aimed to search for neutron EDM by measuring the effect of spin rotation for neutrons traversing non-centrosymmetric crystal is depicted in Fig. 1.

The working system of crystallographic planes is parallel to the entrance face of the crystal. Only neutrons of wavelengths not equal to the Bragg one can pass through the thick working crystal. They are moving in the direction normal to the working planes and undergo reflection from the second crystal (analyzer) whose interplanar spacing is controllable. After that, they are directed to the detector by the semitransparent mirror of pyrolytic graphite.

Neutrons satisfied Bragg's condition for the first crystal are completely reflected by its entrance face and cannot reach the second crystal and so the detector. The neutrons, propagating in the working crystal, are affected by a strong electric field whose value is determined by the deviation parameter from the Bragg condition for this crystal. The analyzer crystal from the same material has the same orientation of planes. Its interplanar spacing can be controlled by changing the temperature. Heating or cooling the analyzer, one can select the neutrons of specific wavelength, which passed through the working crystal in a given electric field of the corresponding sign and strength.

An artificial quartz crystal $140 \times 140 \times 35 \mathrm{~mm}$ in size was used in the experiment. The perfectness of the crystal was studied by the method proposed in [39]. The uniformity of the interplanar spacing was $\Delta d / d<5 \times$ $10^{-6}$ over the whole crystal volume. The parameters of the used (110) plane were $g=2.56 \cdot 10^{10} \mathrm{~m}^{-1}$ and $E_{g}=$ $2 \cdot 10^{8} \mathrm{~V} / \mathrm{cm}[23]$.

\subsubsection{Electric-field strength measuring}

For the case where the deviation from Bragg's conditions is $\Delta_{B} \cong 0.5$, the experimental value obtained for the electric-field strength from the measured angle of neutronspin rotation because of Schwinger interaction is $E_{\exp }=$ $(0.7 \pm 0.1) \times 10^{8} \mathrm{~V} / \mathrm{cm}[37,38,40]$, which agrees with the calculated field strength and with the field strength measured earlier by different methods [23].

The electric field was determined from the angle of spin rotation,

$$
\varphi_{S}=\frac{2}{\hbar c v} \mu \boldsymbol{\sigma} \cdot\left[\mathbf{E}_{g} \times \mathbf{v}\right] L \equiv \frac{2\left(\boldsymbol{\mu} \mathbf{H}_{g}^{S}\right)}{\hbar} \frac{L}{v},
$$




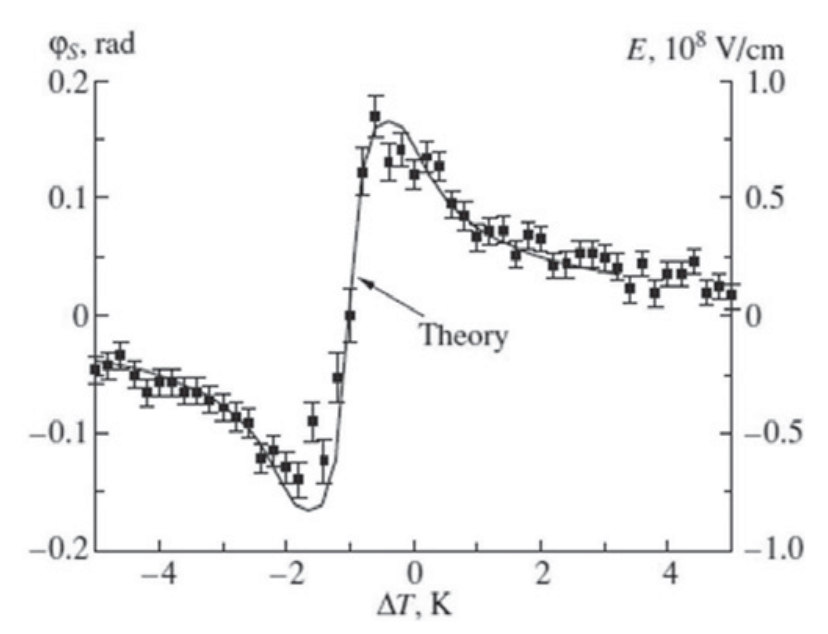

Figure 2. Spin-rotation angle as a function of the temperature difference between the analyzer crystal and the working crystal $[37,38]$.

around the Schwinger magnetic field $\mathbf{H}_{g}^{S}$, where $L$ is the crystal thickness and $\mu$ is the neutron magnetic moment. In order to obtain a nonzero value for the spin-rotation angle $\varphi_{S}$ the crystal turned by an angle of about $4^{\circ}$. So the Bragg angle was $\theta_{B}=86^{\circ}$. In that case $\left[\mathbf{E}_{g} \times \mathbf{v}\right] \neq$ 0 . An example of the experimental dependence of the angle of spin rotation caused by Schwinger interaction on the temperature difference between the analyzer crystal and the working one (that is, on the parameter of deviation from the Bragg condition) is given in Fig. 2. The measurements were performed at the PNPI WWR-M reactor.

Neutrons were propagating through the alpha-quartz crystal of thickness $L=14 \mathrm{~cm}$ at the angle of $86^{\circ}$ with respect to the (110) working plane of the crystal, which is parallel to the entrance face. The respective values of the electric field strength are plotted along the righthand ordinate. The curve has a pronounced resonance character. It is described by the convolution of expression (6) and a finite spectral width of the beam. This width is equal to the Darwin (Bragg) width. The curve passes through zero at $\Delta T \neq 0$. This means that there is a small (at the level of $10^{-5}$ ) but quite measurable difference in interplanar distances between the analyzer crystal that grew under natural conditions and the artificially grown working crystal.

\subsubsection{Test $n E D M$ experiment}

A test experiment devoted to a search for the neutron EDM was performed at the PF1b beam from the ILL reactor $[37,40]$.

The experiment yielded zero result in measuring the angle of neutron-spin rotation around the electric and pseudomagnetic field (reciprocal lattice vector) [40]

$$
\varphi_{g}=(0.9 \pm 2.3) \times 10^{-4} \mathrm{rad} .
$$

The following constraint on the neutron EDM corresponds to this result:

$$
d_{n}<9 \cdot 10^{-24} e \cdot \mathrm{cm}(90 \%)
$$

This constraint is already by two orders of magnitude better than that in Shull's diffractive experiment [17]. The

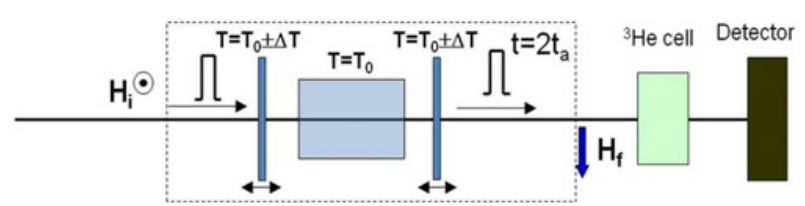

Figure 3. Scheme of the storage variant for the crystal-diffraction nEDM search experiment.

respective constraint on $g_{S} g_{P}$ is

$$
g_{S} g_{P}<6 \cdot 10^{-33} \cdot\left(g^{2}+1 / \lambda_{A}^{2}\right),
$$

where $g^{-1}$ and $\lambda_{A}$ measured in $\mathrm{m}$. From a comparison of the constraints on this quantity from different experiments [41-44], one can see that, in the interaction range of $\lambda_{A}<$ $10^{-7} \mathrm{~m}$, our constraints are the best $[32,36]$.

Test experiments $[37,38,40]$ demonstrated that the sensitivity to measure neutron EDM can be at the level of $2 \cdot 10^{-26} e \cdot \mathrm{cm}$ for the 100 days of statistic accumulation for the available quartz crystal and PF1b cold neutron beam of ILL reactor. Essential advance of the method can be reached by using other crystals, see table below.

Here $\tau_{a}$ is the time of neutron absorption in a crystal. One can see that $E_{g} \cdot \tau_{a}$ for some crystals by an order more than that for quartz crystal. Unfortunately, to reach such a value we need too large crystal dimension, up to a few meters. Below we discuss a way to solve this problem.

\subsubsection{New storage variant of the experiment}

Using a new generation of spallation neutron sources, such as ESS, we proposed a storage variant of the crystal-diffraction nEDM experiment [45] that result is an essential increasing time of neutron interaction with the electric field for rather thin crystal, about $10-20 \mathrm{~cm}$, without losing setup luminosity. The idea of the storage modification of the experiment is shown in a Fig. 3 .

Three crystals are placed to the parallel position. Then the beam pulse comes to the first one it should be slightly moved with the velocity more than Bragg width of the reflex and neutron pulse pass through the crystal. After that neutron pulse will be trapped between the two thin crystals kept with the same temperature. The large crystal placed between the thin ones is kept with a slightly different temperature to get the corresponding value and sign of the electric field. After the time $t$ equal to the double absorption time the third crystal should be moved with the same velocity and neutron pulse come to the detector after polarization analysis. The usual value of Bragg width for cold neutron reflection is $\sim 1 \mathrm{~cm} / \mathrm{s}$, so we should move the crystals with the same velocity that is not a problem.

The preliminary estimations of sensitivity to measure nEDM for storage modification of the setup give a few $10^{-27} e \cdot \mathrm{cm}$ for the designed luminosity of short pulse facility of ESS and crystal of $\mathrm{Bi}_{12} \mathrm{SiO}_{20}$ with dimensions $\sim 10 \times 10 \times 10 \mathrm{~cm}^{3}$ for 100 days of statistic accumulation. The requirements of crystal perfectness are not too strong. The uniformity of the interplanar spacing should be at the level $\Delta d / d<5 \cdot 10^{-6}$ over the whole crystal volume and the angular mosaicity at the level of one angular minute because of wide (tens minutes) angular diffraction width for Bragg angles close to the right one. 
Table 1. Property of some crystals without a center of symmetry.

\begin{tabular}{lcccccc}
\hline Crystal & $\begin{array}{c}\text { Symmetry } \\
\text { group }\end{array}$ & hkl & $\begin{array}{c}d \\
{[\AA]}\end{array}$ & $\begin{array}{c}E_{g} \cdot 10^{-8} \\
{[\mathrm{~V} / \mathrm{cm}]}\end{array}$ & $\begin{array}{c}\tau_{\alpha} \\
{[\mathrm{ms}]}\end{array}$ & $\begin{array}{c}E_{g} \cdot \tau_{\alpha} \\
{[\mathrm{kV} / \mathrm{cm} \cdot \mathrm{s}]}\end{array}$ \\
\hline$\alpha$-quarz & $32\left(\mathrm{D}_{3}^{6}\right)$ & 110 & 2.457 & 2.0 & 1 & 200 \\
$\mathrm{Bi}_{12} \mathrm{SiO}_{20}$ & $\mathrm{I} 23$ & 444 & 1.46 & 4.65 & 4 & 1860 \\
$\mathrm{Bi}_{12} \mathrm{GeO}_{20}$ & $\mathrm{I} 23$ & 444 & 1.46 & 4.8 & 1 & 480 \\
$\mathrm{PbO}$ & $\mathrm{P} \mathrm{c} \mathrm{a} 21$ & 002 & 2.94 & 10.4 & 1 & 1040 \\
$\mathrm{BeO}$ & $6 \mathrm{~mm}$ & 201 & 1.13 & 6.5 & 7 & 4500 \\
\hline
\end{tabular}

\section{Precise neutron spectroscopy}

There is a well-known effect of diffraction enhancement when a small variation of the incident neutron beam direction within Bragg width (a few arc seconds) results in a significant change within the Bragg angle (a few tens degrees) of the neutron flux density direction inside the crystal. This direction can be described by the angle $\Omega$ measured from the crystallographic plane, i.e. from the direction of neutron flux density along the plane for exact Bragg condition. It is equal to

$$
\Omega=\Delta \theta \cdot \frac{E}{v_{g}} \cdot 2 \sin ^{2} \theta_{B},
$$

where $\theta_{B}$ is the Bragg angle, $\Delta \theta$ is the deviation from the $\theta_{B}, E$ is the neutron energy and $v_{g}$ is the amplitude of $g$-harmonic of the periodic neutron-crystal interaction potential. This effect was used [46] in the measurement of neutron refraction by the prism in the double-crystal diffraction pattern. A similar effect for a neutron flux density deflection can be observed also due to variation of the neutron energy because angular deviation from the Bragg condition directly depends on the change $\Delta E$ in neutron energy:

$$
\Delta \theta=\frac{\Delta E}{2 E} \tan \theta_{B}
$$

This feature was used for measurements of neutron refraction in the magnetic field [47].

The two-crystal scheme of Laue diffraction with two crystals placed in magnetic fields of opposite directions was considered in [48]. In this scheme, the crystals are used as "amplifiers" of neutron refraction in the magnetic field. So the magnitude of the effect caused by the influence of an external force acting on a neutron in such scheme is about $K_{g}=E / v_{g}$ times greater than that for standard techniques SESANS. Value $K_{g}$ for the cold neutron diffraction is $\sim 10^{5}$, because of $E \sim 10^{-2} \mathrm{eV}$ and $v_{g} \sim 10^{-7} \mathrm{eV}$.

However, it is more convenient to use the scheme of experiment based on the effect of the diffraction focusing in Laue diffraction [49], see Fig. 4. In such geometry, a part of the diffracted beam will be focused on the exit surface of the second crystal [50] so the intensity distribution, as well as the neutron polarization distribution at the entrance surface of the first crystal, will be reproduced at the exit surface of the second crystal.

We can choose the positions of the coils to make the angles of the neutron spin rotation in the coil $\mathrm{K} 1$ equal to those in the coil $\mathrm{K} 2$, but because of those angles have opposite signs for any neutron trajectory, the total angle

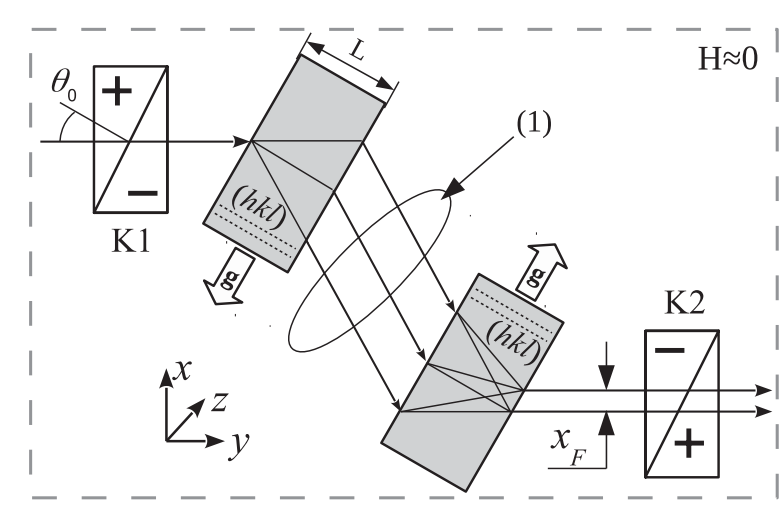

Figure 4. Idea of the experiment using neutron Laue diffraction. $\mathrm{K} 1, \mathrm{~K} 2$ are the coils with the magnetic field $B$, the signs " + " and " - " correspond to the directions of the magnetic field.

of rotation will be zero, see [51]. The presence of external force acting on a neutron between the crystals will lead to the change of neutron direction and to the focus shifts [50]. Due to such shifts, a neutron spin will turn by the angle $\phi_{x}$, because some difference arises of the neutron path lengths in opposite magnetic fields. The angle, corresponding to the focus shift $x$, is equal to

$$
\phi_{x}=\frac{2 \mu B}{\hbar} \cdot \frac{2 x \tan \theta_{0}}{v},
$$

where $v$ is the neutron velocity, $B$ is the value of magnetic fields in the coils $\mathrm{K} 1$ and $\mathrm{K} 2, \theta_{0}$ is the angle between the neutron velocity and the normal to the coils boundary.

If a neutron changes its direction in the area (1) by the angle $\alpha$, the shift of the neutron beam spot at the exit surface of the second crystal will be equal to

$$
x_{\alpha}=\frac{\alpha}{\gamma_{B}} L \tan \theta_{B}=2 \alpha L \sin ^{2} \theta_{B} \frac{E}{v_{g}},
$$

where $\gamma_{B}$ is the Bragg width, $L$ is the crystal thickness. The action of an external force $F_{n}$ at a distance $l$ will change the direction of the neutron velocity by the angle $\alpha=F_{n} l /(2 E)$ and the shift of the beam focus spot at the exit of the second crystal will be

$$
x_{F}=\frac{F_{n} l}{v_{g}} L \sin ^{2} \theta_{B} .
$$

The main parameter, which limits the angular resolution of the method, is the spot size of a spatially focused neutron beam. The beam spreading over the surface of the second crystal by the value of $\Delta x$ will lead to the spreading of angles of neutron spin rotation after the passage the coil $\mathrm{K} 2$ by the value of $\Delta \phi$, see (13). To 


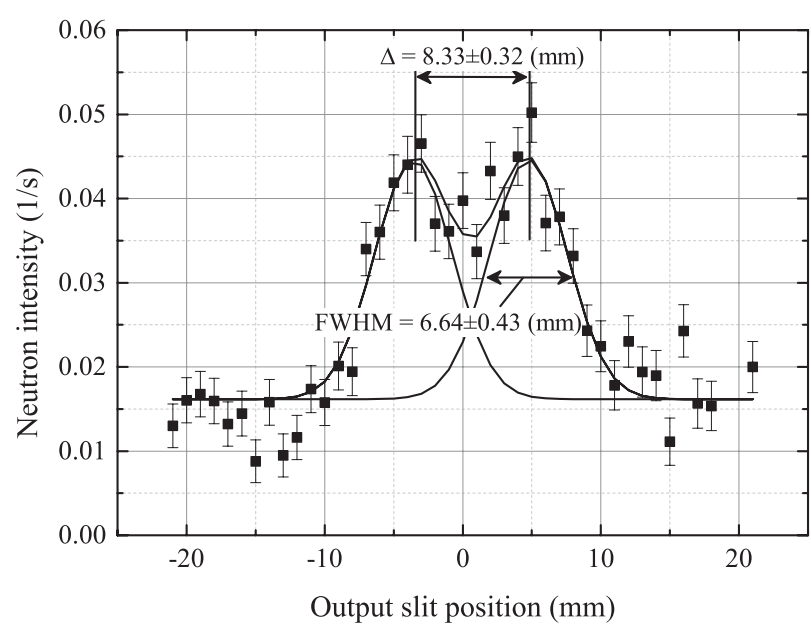

Figure 5. The intensity dependence on the exit slit position. The dimensions of the entrance and exit slits were $4 \mathrm{~mm}, \theta_{B}=68^{\circ}$. Beam aperture in the region (2) is equal to $56 \mathrm{~mm}$.

observe the spin rotation effect the condition $\Delta \phi<2 \pi$ should be satisfied.

The physical limit of spatial resolution in the doublecrystal diffraction pattern is determined by the value [49]:

$$
x_{w}=\frac{\xi_{g} \tan \theta_{B}}{2 \pi}=\frac{V}{4 F_{g} d}
$$

where $\xi_{g}=\pi V /\left(2 F_{g} d \tan \theta_{B}\right)$ is the extinction length, $V$ is the volume of the crystal unit cell, $d$ is the interplanar spacings, $F_{g}$ is the structure factor. The values of $x_{w}$ for cold neutron diffraction are usually of $(10-50) \mu \mathrm{m}$. For instance, for the (220) plane of silicon $x_{w}=26 \mu \mathrm{m}$. Such spatial resolution allows to use the magnetic fields up to $1 \mathrm{kGs}$. As a result, for the silicon crystals with the plane (220) and $10 \mathrm{~cm}$ thicknesses, value of the electric field $E_{0}=100 \mathrm{kV} / \mathrm{cm}$ in the area (1), see. Fig. 4, and $l=1 \mathrm{~m}$, the neutron spin rotation angle (due to presence of the neutron electric charge $e_{n}$ ) will be, see. (13) and (15)

$$
\phi_{e} \simeq 1 \cdot 10^{18} e_{n}
$$

for the Bragg angle $\theta_{B}=70^{\circ}, e_{n}$ is in units of electron charge. Thus, for accuracy to measure the angle of the neutron spin rotation at the level of $\sigma\left(\phi_{e}\right) \sim 10^{-5}$, we can expect the sensitivity to measure the neutron charge at the level of $\sim 10^{-23}$ of the electron charge, which is two orders of magnitude better than the modern constraint [52].

\subsection{Experiment}

As the first stage of this project, we studied the effect of diffraction focusing in the double-crystal scheme of Laue diffraction. The experiment was carried out at the reactor WWR-M (PNPI, Gatchina). Crystals of silicon had the dimensions $110 \times 110 \times 100 \mathrm{~mm}^{3}$ and the working crystallographic plane (220) $(d=1.92 \AA$, $\left.F_{g}=32 \cdot 10^{-13} \mathrm{~cm}, v_{g}=5 \cdot 10^{-8} \mathrm{eV}\right)$. Crystals together with the rotating platform placed in a thermostatic volume. The stability of the crystal temperature was $10^{-2} \mathrm{~K} /$ day. The distance between the crystals was fixed. At the exit face of the second crystal, we had two spots from the direct and reflected beams. An example of the experimentally measured intensity distribution over the exit face of the second crystal is shown in Fig. 5.
The splitting into two components coincided with the calculated value. The estimated own spatial resolution obtained from Fig. 5 was $W_{m}<3$. Unfortunately, the low luminosity of the experiment allows us to give only an upper limit for the $W_{m}$.

The value of the beam displacement along exit surface (5) is determined by angular deviation of the neutron:

$$
x_{\alpha}=2 \cdot 10^{7} \alpha[\mathrm{mm}] .
$$

So, the spatial resolution $W_{m}<3 \mathrm{~mm}$ corresponds to the angular one $\alpha_{W}<1.5 \cdot 10^{-7}=0.03^{\prime \prime}$.

\section{Conclusion}

Strong intracrystalline fields (up to $10^{9} \mathrm{~V} / \mathrm{cm}$ ), acting on a neutron in non-centrosymmetric crystal, give rise for a new method of a search for the neutron EDM. An accuracy of this method is expected at the level $\sim 2 \cdot 10^{-26} e \cdot \mathrm{cm}$ for available quartz crystals and can reach about $\sim 2 \cdot 10^{-27} e \cdot \mathrm{cm}$ for the BSO crystal and the "storage" variant of the setup. Such sensitivity also allows getting a best direct constraint on the parameters of hypothetic CP-violating short-range nucleon-nucleon interaction for the range $\lambda<10^{-6} \mathrm{~m}$.

The first experimental results obtained at the prototype of two-crystal setup confirmed the possibility to develop ultra-precise neutron spectroscopy, due to significant enhancement of an external force acting on a neutron, using Laue diffraction in perfect crystals under Bragg angles close to the right one. The proposed ultra-precise technique can used for a broad range of experiments, such as measurements of the neutron electric charge; a test of the equivalence principle; measurements of the neutron scattering amplitudes and study of the quality of crystals.

This work was supported by Ministry of Science and Education of the Russian Federation (program 3.3838.2017/4.6).

\section{References}

[1] M. Pospelov, A. Ritz, Annals of Phys. 318, 119 (2005)

[2] I.S. Altarev, et al., Yad. Fiz. 59, 1204 (1996)

[3] C.A. Baker, et al., Phys. Rev. Lett. 97, 131801 (2006)

[4] J.M. Pendlebury, et al., Phys. Rev. A 70, 032102 (2004)

[5] A.P. Serebrov, et al., NIM A 611, 276 (2009)

[6] O. Zimmer, F.M. Piegsa, S.N. Ivanov, Phys. Rev. Lett. 107, 13480 (2011)

[7] A.P. Serebrov, et al., Phys. Rev. C 92, 055501 (2015)

[8] C.A. Baker, et al., Phys. Proc. 17, 159 (2011)

[9] C. Abel, et al. (nEDM collaboration), EPJ Web of Conf. 219, 02001 (2019)

[10] I. Altarev, et al., Nuovo Cim. C035N04, 122 (2012)

[11] A. Kolarkar, AIP Conf. Proc. 1200, 861 (2010)

[12] A. Saunders, et al., Rev. Sci. Instrum. 84, 013304 (2013)

[13] K. Matsuta et al., AIP Conf. Proc. 1560, 152 (2013)

[14] F.M. Piegsa, Phys. Rev. C 88, 045502 (2013)

[15] S.K. Lamoreaux, R. Golub, J. Phys. G 36, 104002 (2009)

[16] P. Schmidt-Wellenburg, AIP Conf. Proc. 1753, 060002 (2016) [arXiv:1607.06609v2] 
[17] C.G. Shull, R. Nathans, Phys. Rev. Lett. 19, 384 (1967)

[18] Yu.G. Abov, A.D. Gulko, P.A. Krupchitsky, Polarized Slow Neutrons (Atomizdat, Moscow, 1966), p. 256 (in Russian)

[19] M. Forte, J. Phys. G 9, 745 (1983)

[20] V.G. Baryshevskii, S.V. Cherepitsa, Phys. Stat. Sol. B 128379 (1985)

[21] V.G. Baryshevskii, S.V. Cherepitsa, Izvestiya Vuzov SSSR, Ser. Fiz. 8, 110 (1985) (in Russian)

[22] V.L. Alekseev, et al., JETP 67, 1727 (1988)

[23] V.L. Alexeev, et al., NIM A 284, 181 (1989); V.L. Alexeev, et al., JETP 69, 1083 (1989)

[24] M. Forte, C.M.E. Zeyen, NIM A 284, 147 (1989)

[25] V.V. Fedorov, et al., J. Phys. G 18, 1133 (1992)

[26] V.V. Fedorov, et al., Tech. Phys. Lett. 21, 881 (1995)

[27] V.V. Fedorov, et al., Tech. Phys. Lett. 21, 884 (1995)

[28] V.V. Fedorov, et al., Phys. B 234-236, 8 (1997)

[29] V.V. Fedorov, et al., Phys. B 297, 293 (2001)

[30] V.V. Fedorov, V.V. Voronin, NIM B 201, 230 (2003)

[31] V.V. Fedorov, et al., JETP Lett. 80, 675 (2004)

[32] V.V. Fedorov, V.V. Voronin, Phys. Atomic Nucl. 77, 695 (2014)
[33] V.V. Fedorov, XXVI Winter LNPI School, vol. 1 (Leningrad, 1991), p. 65

[34] E. Leli ‘evre-Berna, et al., Physica B 397, 120 (2007)

[35] J. E. Moody and F. Wilczek, Phys. Rev. D 30, 130 (1984)

[36] V.V. Voronin, et al., JETP Lett. 90, 5 (2009)

[37] V.V. Fedorov, et al., NIM A 611, 124 (2009)

[38] V.V. Fedorov, et al., NIM, B 252, 131 (2006)

[39] V. V. Fedorov, et al., NIM A 593, 472 (2008)

[40] V.V. Fedorov, et al., Phys. Lett. B 694, 22 (2010)

[41] S. Baessler, et al., Phys. Rev. D 75, 075006 (2007)

[42] A.P. Serebrov. Phys. Lett. B 680, 423 (2009)

[43] A.P. Serebrov, et al., JETP Lett. 91, 6 (2010)

[44] A. Petukhov, et al., Phys. Rev. Lett. 105, 170401 (2010)

[45] V.V. Fedorov, et al., Physica B 406, 2370 (2011)

[46] S. Kikuta, et al., J. Phys. Soc. Japan 39, 471 (1975)

[47] A. Zeilinger, C.G. Shull, Phys. Rev. B 19, 3957 (1979)

[48] V.V. Voronin, et al., Physics Procedia 42, 25 (2013)

[49] V.L. Indenbom, et al., JETP, 39, 542 (1974)

[50] J. Arthur, et al., Phys. Rev. 32, 5753 (1985)

[51] Yu.V. Borisov, et al., Tech. Phys. Lett. 41, 867 (2015)

[52] J. Baumann, et al., Phys. Rev. D 37, 3107 (1988) 